\title{
INVERSE JACOBI MULTIPLIERS AND FIRST INTEGRALS FOR NONAUTONOMOUS DIFFERENTIAL SYSTEMS
}

\author{
ADRIANA BUICA $\breve{1}^{1} \&$ ISAAC A. GARCÍA ${ }^{2}$
}

\begin{abstract}
In this paper we consider nonautonomous differential systems of arbitrary dimension and first find expressions for their inverse Jacobi multipliers and first integrals in some nonautonomous invariant set in terms of the solutions of the differential system. Given an inverse Jacobi multiplier $V$, we find a relation between the Poincaré translation map $\Pi$ at time $T$ that extends to arbitrary dimensions the fundamental relation for scalar equations, $V(T, \Pi(x))=V(0, x) \Pi^{\prime}(x)$, found in Trans. Amer. Math. Soc. 362 (2010), 3591-3612. The main result guarantees the existence of continua of $T$-periodic solutions for $T$-periodic systems in the presence of $T$-periodic first integrals and inverse Jacobi multipliers.
\end{abstract}

\section{INTRODUCTION}

We consider a nonautonomous differential system

$$
\dot{x}=f(t, x),
$$

where $f: I \times \tilde{U} \rightarrow \mathbb{R}^{n}$ is a $\mathcal{C}^{1}$ function, $I \subset \mathbb{R}$ is an open interval, $\tilde{U} \subset \mathbb{R}^{n}$ is open. A solution of (1) is a $\mathcal{C}^{1}$ function $\varphi: J \rightarrow \tilde{U}$ that satisfies the equation for all $t$ in the open interval $J \subset I$. We denote, as usual, the components of the column vectors $x=\left(x_{1}, \ldots, x_{n}\right)$ and $f=\left(f_{1}, \ldots, f_{n}\right)$. We associate to system (1) the vector field

$$
\mathcal{X}=\partial_{t}+\sum_{i=1}^{n} f_{i}(t, x) \partial_{x_{i}} .
$$

The divergence of $\mathcal{X}$ is $\operatorname{div} \mathcal{X}=\sum_{i=1}^{n} \partial f_{i} / \partial x_{i}$.

Definition 1. A function $V: \Omega \rightarrow \mathbb{R}$ is said to be an inverse Jacobi multiplier for system (1) in the open set $\Omega \subset I \times \tilde{U}$ if $V$ is of class $\mathcal{C}^{1}(\Omega)$, it is not locally null and it satisfies the following linear first order partial differential equation:

$$
\mathcal{X} V=V \operatorname{div} \mathcal{X}
$$

We remark that in the special cases of autonomous planar systems and, respectively nonautonomous scalar equations, instead of the term inverse Jacobi

2010 Mathematics Subject Classification. 34A34, 34C25, 35F10, 70H06, 70H12.

Key words and phrases. non-autonomous systems, inverse Jacobi multipliers, Poincaré translation map, periodic solutions.

The authors are partially supported by a MCYT/FEDER grant number MTM2008-00694 and by a CIRIT grant number 2014 SGR 1204. 
multiplier it is commonly used the term inverse integrating factor.

Jacobi introduced his (last) multiplier $1 / V$ for the first time in the work [12] in 1844, for the sole purpose of finding the (last) additional first integral taking into account that equation (2) is equivalent to the divergence free condition $\operatorname{div}(\mathcal{X} / V) \equiv 0$. Since then, inverse Jacobi multipliers (inverse integrating factors) have been used in order to study autonomous planar systems resulting interesting and powerful properties (see, for example, the survey [8]). For the use of inverse Jacobi multipliers in the study of higher dimensional systems (mainly autonomous) we remind here only the survey [2] and the recent work [3], and we emphasize that the literature is not so rich as in the case of planar systems. One of the specific subjects where inverse Jacobi multipliers have been proved to be especially useful is related to closed orbits of autonomous systems: limit cycles or continua of periodic solutions.

When studying planar autonomous systems via inverse integrating factors, sometimes the authors need to transform the system and the inverse integrating factor into a nonautonomous scalar equation and, respectively its corresponding nonautonomous inverse integrating factor. This is the case in $[6,9,10]$. We were motivated by these works to study nonautonomous scalar equations and their inverse integrating factors, and, in fact, we gave results for nonautonomous systems of arbitrary dimension.

In Theorem 3 of [9], passing to curvilinear coordinates, a planar autonomous system is transformed in a neighborhood of a regular orbit into some scalar (thus with $n=1$ ) nonautonomous equation of the form (1). The authors prove a fundamental relation which is a differential equation for the Poincaré map written in terms of an inverse integrating factor. This relation proved to be useful in analyzing the bifurcation of limit cycles from a multiple limit cycle as well as from a homoclinic orbit in [9]. In this work we find an analogous of this fundamental relation for nonautonomous systems of arbitrary dimension. The result is stated below.

First we introduce some notation used throughout this work. For each $\left(t_{0}, y\right) \in$ $I \times \tilde{U}$ we denote by $\psi\left(\cdot ; t_{0}, y\right)$ the solution of $(1)$ satisfying $\psi\left(t_{0} ; t_{0}, y\right)=y$ and by $I_{\left(t_{0}, y\right)}$ its maximal interval of existence. Moreover, we recall that the Poincaré translation map at time $\tau>0$ is the map $\Pi: U \subset \tilde{U} \rightarrow \tilde{U}$ defined by $\Pi(x)=$ $\psi(\tau ; 0, x)$ where $U$ is such that $[0, \tau] \subset I_{(0, x)}$ for any $x \in U$.

Theorem 2. Let $V: \Omega \rightarrow \mathbb{R}$ be an inverse Jacobi multiplier of (1). If there exists some $\tau>0$ and some open set $U \subset \tilde{U}$ such that $[0, \tau] \subset I_{(0, x)}$ and $(t, \psi(t ; 0, x)) \in$ $\Omega$ for all $t \in[0, \tau]$ and $x \in U$, the following relation holds

$$
V(\tau, \Pi(x))=V(0, x) \operatorname{det} D \Pi(x), \quad \text { for all } x \in U .
$$

A key ingredient in the proof of one of the main results (Theorem 1.2) of [6] uses this fundamental relation (3) when $n=1$. In [10] the same relation was used to study multiple Hopf bifurcation in a neighborhood of certain singular point of focus type of a planar autonomous system by means of generalized polar 
coordinates. Moreover, in $[4,14]$, the above Theorem 2 was used to study multiple Hopf bifurcation in a neighborhood of a Hopf point of systems in arbitrary dimensions. We use this result here in order to prove our main result Theorem $3)$.

Both in [9] and [10] appears in a natural way that the corresponding scalar differential equation of the form (1) is also periodic and the inverse integrating factor of the planar system is transformed into a nonautonomous inverse integrating factor which is periodic.

We were motivated by these facts to study periodic inverse Jacobi multipliers for periodic systems (1) of arbitrary dimension. In this context, it is natural to assume what we will call Hypothesis $*$ in the rest of the paper.

Hypotheses *:

(a) System (1) is well defined for any time, that is, $I=\mathbb{R}$;

(b) System (1) is $T$-periodic for some fixed period $T>0$. This means that the function $f(\cdot, x)$ is $T$-periodic for each $x \in \tilde{U}$;

(c) There is an open set $U \subset \tilde{U}$ such that $[0, T] \subset I_{(0, x)}$ for any $x \in U$.

Our main result is stated below. Our contribution is part (ii) and for completeness we state also (i) which is known by specialists.

Theorem 3. Assume Hypothesis * and that there exists $\tilde{x} \in U$ such that $\psi(\cdot ; 0, \tilde{x})$ is a T-periodic solution of (1).

(i) If there exist $n$ independent first integrals in $\mathbb{R} \times \tilde{U}$ of (1) which are $T$ periodic, then there exists an open neighborhood $U_{0}$ of $\tilde{x}$ such that $\psi(\cdot ; 0, x)$ is T-periodic for any initial condition $x \in U_{0}$.

(ii) If there exist $n-1$ independent first integrals, and an inverse Jacobi multiplier $\tilde{V}$ in $\mathbb{R} \times \tilde{U}$ which are T-periodic and such that $\tilde{V}(0, x) \neq 0$ for all $x \in \tilde{U}$, then the T-periodic solution $\psi(\cdot ; 0, \tilde{x})$ is included into a 1-parameter family of $T$-periodic solutions $\psi\left(t ; 0, x^{*}(\mu)\right)$, where $x^{*}$ is a $\mathcal{C}^{1}$ function in some open interval of reals.

The notion of independence for first integrals is the usual one (can be found also in Section 2). A key step in the proof of Theorem 3 (ii) is the fundamental relation (3). We also prove in the forthcoming Theorem 24 the $T$-periodicity of any first integral and any inverse Jacobi multiplier of a system whose solutions are $T$-periodic. We note here that in [7] are given sufficient conditions to ensure the existence of periodic first integrals for Hamiltonian systems of Lie type.

Our paper is organized as follows. In Section 2 we present the proof of Theorem 2 and we also find in Proposition 7, using the characteristics method, the expressions for an inverse Jacobi multiplier and a first integral in some invariant nonautonomous set, as well as a relation between these two objects. Section 3 contains the above mentioned Theorems 3 and 24 on $T$-periodic systems together with some useful examples.

In this paper det denotes the determinant, $D$ is the symbol for the derivative (the Jacobian matrix if applied to a vector function of various variables), $D_{x}$ is 
the symbol for the derivative with respect to $x, \nabla$ is the symbol for the gradient of a real function of various variables.

\section{INVERSE JACOBI MULTIPLIERS AND FIRST INTEGRALS}

Let $U \subset \tilde{U}$ be open. We fix some $t^{*} \in I$, but, for simplicity we write $t^{*}=0$. Let

$$
\Omega^{*}=\left\{(t, \psi(t ; 0, x)) \in I \times \tilde{U}: x \in U, t \in I_{(0, x)}\right\} .
$$

This set is an invariant nonautonomous set in the sense of [13] for the differential equation (1).

In the next lemma we state some properties of a process $\psi\left(t ; t_{0}, x\right)$ and we also write an equivalent definition of the set $\Omega^{*}$ defined in (4).

Lemma 4. Assume that $f \in C^{m}\left(I \times \tilde{U}, \mathbb{R}^{n}\right)$ with $m \in \mathbb{N}^{*} \cup\{\infty\}$. Then:

(i) $\psi: \mathcal{D} \rightarrow \tilde{U}$ is of class $C^{m}$ on $\mathcal{D}=\left\{\left(t, t_{0}, y\right) \in I \times I \times \tilde{U}: t \in I_{\left(t_{0}, y\right)}\right\}$.

(ii) $\Omega^{*}=\left\{(t, y) \in I \times \tilde{U}: 0 \in I_{(t, y)}, \psi(0 ; t, y) \in U\right\}$ and is open.

(iii) $\psi(s ; t, \psi(t ; 0, x))=\psi(s ; 0, x)$ for all $x \in U, t \in I_{(0, x)}, 0 \leq s \leq t$.

(iv) $\psi(t ; 0, \psi(0 ; t, y))=y$ for all $(t, y) \in \Omega^{*}$.

Proof. Statement (i) is Theorem 9.5 from [1]. Statement (ii) follows directly from the definition of $\Omega^{*}$. Statements (iii) and (iv) express the cocycle property of a process of a nonautonomous differential equation that is a consequence of the fact that the solutions are determined uniquely by their initial values. For insight in these topic one can see $[11,13]$.

REMARK 5. Relation (iv) in Lemma 4, which can be written as

$$
y=\psi(t ; 0, x) \text { if and only if } \quad x=\psi(0 ; t, y)
$$

is a key ingredient in the proof of many statements in this paper.

Proof of Theorem 2. Let $x \in U$ be fixed. We denote $\varphi(t)=V(t, \psi(t ; 0, x))$ and $a(t)=\operatorname{div} \mathcal{X}(t, \psi(t ; 0, x))$ for any $t \in[0, \tau]$. Using that $V$ satisfies the linear partial differential equation (2), i.e. $\mathcal{X} V=V \operatorname{div} \mathcal{X}$, we obtain

$$
\dot{\varphi}(t)=(\mathcal{X} V)(t, \psi(t ; 0, x))=\operatorname{div} \mathcal{X}(t, \psi(t ; 0, x)) V(t, \psi(t ; 0, x))=a(t) \varphi(t) .
$$

Then $\varphi(t)=\varphi(0) \exp \left[\int_{0}^{t} a(s) d s\right]$ which gives for $x \in U$ and $t \in[0, \tau]$,

$$
V(t, \psi(t ; 0, x))=V(0, x) \exp \left[\int_{0}^{t} \operatorname{div} \mathcal{X}(s, \psi(s ; 0, x)) d s\right] .
$$

On the other hand, the matrix function $D_{x} \psi(\cdot ; 0, x)$ is the principal fundamental matrix solution of the linear variational system $\dot{u}=D_{x} f(t, \psi(t ; 0, x)) u$. Liouville's formula (see for example page 152 of [5]) written for this linear system is

$$
\operatorname{det}\left(D_{x} \psi(t ; 0, x)\right)=\exp \left[\int_{0}^{t} \operatorname{div} \mathcal{X}(s, \psi(s ; 0, x)) d s\right], \quad t \in I_{(0, x)}, x \in U .
$$


Using (5) and (6) we obtain

$$
V(t, \psi(t ; 0, x))=V(0, x) \operatorname{det}\left(D_{x} \psi(t ; 0, x)\right), \quad t \in[0, \tau], x \in U .
$$

Relation (3) is obtained taking $t=\tau$ in (7).

We remind the definition of a first integral.

Definition 6. A function $H: \Omega \rightarrow \mathbb{R}$ is said to be a first integral in the open set $\Omega \subset I \times \tilde{U}$ for system (1) if $H$ is of class $\mathcal{C}^{1}(\Omega)$, it is not locally constant and it satisfies the following linear first order partial differential equation:

$$
\mathcal{X} H=0 .
$$

In the next proposition it is shown how, with a given initial condition, one can build the explicit expression of an inverse Jacobi multiplier and a first integral of (1) in the nonautonomous invariant set $\Omega^{*}$ in terms of the associated process $\psi\left(t ; t_{0}, y\right)$. Parts (i) and (ii) of this result are well-known, but we present it here for completeness.

Proposition 7. The following statements hold.

(i) Let $\Omega \subset I \times \tilde{U}$ be open and assume that there exists an inverse Jacobi multiplier $\tilde{V}: \Omega \rightarrow \mathbb{R}$ of $(1)$ such that $\tilde{V}(t, x) \neq 0$ for any $(t, x) \in \Omega$. We have that $V: \Omega \rightarrow \mathbb{R}$ is an inverse Jacobi multiplier of (1) if and only if there exists a first integral $H$ (or a constant) of (1) in $\Omega$ such that

$$
V=H \tilde{V} \text {. }
$$

(ii) Let $F: U \rightarrow \mathbb{R}$ be a $\mathcal{C}^{1}$ function. We have that $H: \Omega^{*} \rightarrow \mathbb{R}$ is a first integral of (1) with $H(0, \cdot)=F$ if and only if

$$
H(t, y)=F(\psi(0 ; t, y)), \quad(t, y) \in \Omega^{*} .
$$

(iii) Let $V: \Omega^{*} \rightarrow \mathbb{R}$ be an inverse Jacobi multiplier of system (1) and denote $F=V(0, \cdot)$. Then

$$
V(t, y)=F(\psi(0 ; t, y)) V^{*}(t, y), \quad(t, y) \in \Omega^{*},
$$

where $V^{*}: \Omega^{*} \rightarrow \mathbb{R}$ has the expressions

$$
V^{*}(t, y)=\exp \left[\int_{0}^{t} \operatorname{div} \mathcal{X}(s, \psi(s ; t, y)) d s\right]=\operatorname{det} D_{x} \psi(t ; 0, \psi(0 ; t, y)) .
$$

If $V^{*} \in \mathcal{C}^{1}\left(\Omega^{*}\right)$ (this is assured when $f$ is $C^{2}$ ) then $V^{*}$ is an inverse Jacobi multiplier of (1) and, moreover, given $F \in \mathcal{C}^{1}(U), V: \Omega^{*} \rightarrow \mathbb{R}$ is an inverse Jacobi multiplier of system (1) with $V(0, \cdot)=F$ if and only if (9) holds.

Proof. (i) Let $V: \Omega \rightarrow \mathbb{R}$ be an inverse Jacobi multiplier of (1). Then $V / \tilde{V} \in$ $\mathcal{C}^{1}(\Omega)$ and, by direct calculations using the relations $\mathcal{X} V=V \operatorname{div} \mathcal{X}$ and $\mathcal{X} \tilde{V}=$ $\tilde{V} \operatorname{div} \mathcal{X}$, one can obtain that $\mathcal{X}(V / \tilde{V})=0$. Thus $H=V / \tilde{V}$ is a first integral of (1) in $\Omega$. 
Conversely, now let $H: \Omega \rightarrow \mathbb{R}$ be a first integral. Then $H \tilde{V} \in \mathcal{C}^{1}(\Omega)$ and, using the relations $\mathcal{X} H=0$ and $\mathcal{X} \tilde{V}=\tilde{V} \operatorname{div} \mathcal{X}$, one can obtain that $\mathcal{X}(H \tilde{V})=$ $(H \tilde{V}) \operatorname{div} \mathcal{X}$. Thus $V=H \tilde{V}$ is indeed an inverse Jacobi multiplier of (1) in $\Omega$.

(ii) Let first $H: \Omega^{*} \rightarrow \mathbb{R}$ be a first integral with $H(0, x)=F(x)$ for any $x \in U$. Using that $\mathcal{X} H=0$ in $\Omega^{*}$, one can see that the derivative with respect to $t$ of $H(t, \psi(t ; 0, x))$ is zero for all $t \in I_{(0, x)}$. Hence $H(t, \psi(t ; 0, x))=H(0, \psi(0 ; 0, x))=$ $H(0, x)=F(x)$ for all $t \in I_{(0, x)}$ and $x \in U$. Taking $x=\psi(0 ; t, y)$ for $(t, y) \in \Omega^{*}$ and using (iv) of Lemma 4 we obtain the conclusion.

Conversely, let now $H(t, y)=F(\psi(0 ; t, y))$ for all $(t, y) \in \Omega^{*}$. Using again Lemma 4 one can see that $H$ satisfies for any $x \in U$ and $t \in I_{(0, x)}, H(t, \psi(t ; 0, x))=$ $F(x)$. Taking the derivative with respect to $t$ we obtain that $H$ satisfies $\mathcal{X} H=0$ in $\Omega^{*}$.

(iii) Like in the proof of Theorem 2 one can show that $V$ satisfies (5) for any $x \in U$ and $t \in I_{(0, x)}$. In (5) we put $F(x)$ instead of $V(0, x)$, and $x=\psi(0 ; t, y)$ for $(t, y) \in \Omega^{*}$ such that finally we obtain (9) with $V^{*}$ having the first expression given in the statement. In order to obtain the second expression of $V^{*}$ we replace $x=\psi(0 ; t, y)$ for $(t, y) \in \Omega^{*}$ in $(6)$.

Finally assume that $V^{*} \in \mathcal{C}^{1}\left(\Omega^{*}\right)$ and note that, taking in its expression $y=$ $\psi(t ; 0, x)$, for any $x \in U$ and $t \in I_{(0, x)}$ we obtain

$$
V^{*}(t, \psi(t ; 0, x))=\exp \left[\int_{0}^{t} \operatorname{div} \mathcal{X}(s, \psi(s ; 0, x)) d s\right] .
$$

Taking the derivative with respect to the variable $t$ in (10) one obtains the relation $\mathcal{X} V^{*}=V^{*} \operatorname{div} \mathcal{X}$ which holds true in any point of the form $(t, \psi(t ; 0, x))$, hence in any $(t, y) \in \Omega^{*}$. Now let $F \in \mathcal{C}^{1}(U)$. Since, by (ii), $F(\psi(0 ; t, y))$ is a first integral, and $V^{*}$ is an inverse Jacobi multiplier in $\Omega^{*}$, applying (i) we obtain that $V$ given by (9) is indeed an inverse Jacobi multiplier.

In the next example we see the expression of $V^{*}$ (given in Proposition 7 (iii)) for a linear system.

Example 8. Let $A \in C\left(\mathbb{R}, \mathbb{R}^{n \times n}\right)$ and $b \in C\left(\mathbb{R}, \mathbb{R}^{n}\right)$. For a linear differential system $\dot{x}=A(t) x+b(t)$ we have that the inverse Jacobi multiplier $V^{*}$ is given by $V^{*}(t, x)=\exp \left(\int_{0}^{t} \operatorname{Tr}(A(s)) d s\right)$, for all $(t, x) \in \mathbb{R} \times \mathbb{R}^{n}$ where $\operatorname{Tr}(A(s))$ is the trace of $A(s)$. We can choose here $U=\mathbb{R}^{n}$ and find that $\Omega^{*}=\mathbb{R} \times \mathbb{R}^{n}$.

Now, for the simple scalar equation $\dot{x}=x^{3}$, we present its family of timedependent inverse Jacobi multipliers. This is just to see how Proposition 7 works. Anyway, in some problems it is important to know the expression of an inverse Jacobi multiplier of some system in order to study perturbations of that system (see $[9,10,4,14]$.

EXAMPLE 9. We consider the differential equation $\dot{x}=x^{3}$ in $\mathbb{R}^{2}$. For any $t_{0}, x \in$ $\mathbb{R}$ we have $\psi\left(t ; t_{0}, x\right)=x / \sqrt{1-2\left(t-t_{0}\right) x^{2}}, I_{\left(t_{0}, x\right)}=\left(-\infty, t_{0}+1 /\left(2 x^{2}\right)\right)$ for $x \neq 0$ 
and $I_{\left(t_{0}, 0\right)}=\mathbb{R}$. Choosing $U=\mathbb{R}$ one can find that $\Omega^{*}=\left\{(t, x) \in \mathbb{R}^{2}: 1+2 t x^{2}>\right.$ $0\}$ and, for an inverse Jacobi multiplier $V$ in $\Omega^{*}$ with $F=V(0, \cdot)$ we have

$$
V(t, x)=F\left(x / \sqrt{1+2 t x^{2}}\right)\left(1+2 t x^{2}\right)^{3 / 2}, \quad(t, x) \in \Omega^{*} .
$$

If we take, for example, $F_{1}(x)=x$ or $F_{2}(x)=x^{3}$ for all $x \in \mathbb{R}$ we obtain two inverse Jacobi multipliers $V_{1}(t, x)=x+2 t x^{3}$ and $V_{2}(x)=x^{3}$, respectively. Note that they are defined in the whole $\mathbb{R}^{2}$ and they satisfy (2) in the whole $\mathbb{R}^{2}$. Here (2) is $\partial V / \partial t+x^{3} \partial V / \partial x=3 x^{2} V$.

The following is the usual definition of a set of $\mathcal{C}^{1}$ (functionally) independent real functions, see for example page 436 of [5]. We consider it for a set of first integrals of (1) and we will use it in the next Section.

Definition 10. Let $1 \leq k \leq n$ and $\Omega \subset I \times \tilde{U}$ be open. We say that $k$ first integrals $H_{1}, \ldots, H_{k}$ of (1) are independent in $\Omega$ if the gradient vectors $\nabla H_{1}(t, y), \ldots, \nabla H_{k}(t, y)$ are linearly independent for each $(t, y) \in \Omega$.

In the next proposition we will see that $n$ first integrals $H_{1}, \ldots, H_{n}$ of (1) are independent in $\Omega^{*}$ if and only if the map $\tilde{F}: U \rightarrow \mathbb{R}^{n}$ defined by $\tilde{F}=$ $\left(H_{1}(0, \cdot), \ldots, H_{n}(0, \cdot)\right)$ is a local diffeomorphism. This result must be known, we state and prove it for completeness and also for further use.

Proposition 11. The set of $n$ first integrals $H_{1}, \ldots, H_{n}$ of $(1)$ are independent in $\Omega^{*}$ if and only if the $\mathcal{C}^{1}$ function $\tilde{F}=\tilde{H}(0, \cdot): U \rightarrow \mathbb{R}^{n}$, where $\tilde{H}=\left(H_{1}, \ldots, H_{n}\right)$, is such that $\operatorname{det} D \tilde{F}(x) \neq 0$ for all $x \in U$.

Proof. We take $n$ first integrals $H_{1}, \ldots, H_{n}$ which are independent in $\Omega^{*}$ and consider $\tilde{F}$ and $\tilde{H}$ like in the statement of this Proposition. Using Proposition 7 (ii) we must have

$$
\tilde{H}(t, y)=\tilde{F}(\psi(0 ; t, y)),(t, y) \in \Omega^{*} .
$$

In order to prove that the set of vectors $\nabla H_{1}(t, y), \ldots, \nabla H_{n}(t, y)$ from $\mathbb{R}^{n+1}$ are linearly independent for each $(t, y) \in \Omega^{*}$, it is necessary and sufficient to prove that the $n \times(n+1)$ matrix $D \tilde{H}(t, y)$ has constant rank $n$ in $\Omega^{*}$. Since using (8) we obtain $\partial \tilde{H} / \partial t=-D_{y} \tilde{H} f$, we deduce that $D \tilde{H}(t, y)$ has constant rank $n$ in $\Omega^{*}$ if and only if $\operatorname{det} D_{y} \tilde{H}(t, y) \neq 0$ for all $(t, y) \in \Omega^{*}$. But using the chain rule and (11) we get $D_{y} \tilde{H}(t, y)=D \tilde{F}(\psi(0 ; t, y)) D_{y} \psi(0 ; t, y)$. Using Liouville's formula like in the proof of Theorem 2 , $\operatorname{det} D_{y} \psi(0 ; t, y)=\exp \left(\int_{t}^{0} \operatorname{div} \mathcal{X}(s, \psi(s ; t, y) d s) \neq 0\right.$. Hence $\operatorname{det} D_{y} \tilde{H}(t, y) \neq 0$ for all $(t, y) \in \Omega^{*}$ if and only if $\operatorname{det} \tilde{F}(\psi(0 ; t, y)) \neq 0$ for all $(t, y) \in \Omega^{*}$, which is equivalent to $\operatorname{det} \tilde{F}(x) \neq 0$ for all $x \in U$.

For our next result we will need the following stronger notion of independence for $n$ first integrals of (1). This notion essentially requires that the local diffeomorphism $\tilde{F}: U \rightarrow \tilde{F}(U)$ defined in Proposition 11 to be actually a global diffeomorphism. This justifies the use of the term globally in the following definition. 
Definition 12. We say that $n$ first integrals $H_{1}, \ldots, H_{n}$ of (1) in $\Omega^{*}$ are globally independent if $\tilde{F}=\tilde{H}(0, \cdot): U \rightarrow \mathbb{R}^{n}$ is a diffeomorphism onto its image, where $\tilde{H}=\left(H_{1}, \ldots, H_{n}\right)$.

Corollary 13. Assume that $f \in C^{m}\left(I \times \tilde{U}, \mathbb{R}^{n}\right)$ with $m \in \mathbb{N}^{*} \cup\{\infty\}$. Then there are $n$ first integrals of (1) in $\Omega^{*}$ of class $C^{m}\left(\Omega^{*}\right)$ which are globally independent.

Proof. Take any diffeomorphism $\tilde{F}: U \rightarrow \tilde{F}(U)$ of class $C^{m}$ and define the function $\tilde{H}: \Omega^{*} \rightarrow \mathbb{R}^{n}$ by $\tilde{H}(t, y)=\tilde{F}(\psi(0 ; t, y))$. Then using Lemma 4 (i) we get that $\tilde{H} \in C^{m}\left(\Omega^{*}, \mathbb{R}^{n}\right)$ and by Proposition 7 (ii) we obtain that its components are first integrals of $(1)$ in $\Omega^{*}$. Clearly, these first integrals are globally independent in $\Omega^{*}$ by construction since $\tilde{F}=\tilde{H}(0, \cdot)$.

As is well-known, independent first integrals can be used to describe any other first integral or inverse Jacobi multiplier. We state below this idea for the $n$ globally independent first integrals given by Corollary 13 .

Proposition 14. Assume that $f \in C^{m}\left(I \times \tilde{U}, \mathbb{R}^{n}\right)$ with $m \in \mathbb{N}^{*} \cup\{\infty\}$. Let $H_{1}, \ldots, H_{n}$ be $C^{m}$ first integrals of $(1)$ in $\Omega^{*}$ which are globally independent. Define the vector function $\tilde{H}=\left(H_{1}, \ldots, H_{n}\right)$. Then we have.

(i) $H: \Omega^{*} \rightarrow \mathbb{R}$ is a $C^{m}$ first integral of (1) if and only if there exists a $C^{m}$ function $\phi$ which is not locally constant and such that $H=\phi(\tilde{H})$ on $\Omega^{*}$.

(ii) Let $m \geq 2$ and $V^{*}: \Omega^{*} \rightarrow \mathbb{R}$ as given in Proposition 7, i.e.

$$
V^{*}(t, y)=\exp \left[\int_{0}^{t} \operatorname{div} \mathcal{X}(s, \psi(s ; t, y)) d s\right] .
$$

$V: \Omega^{*} \rightarrow \mathbb{R}$ is a $C^{m-1}$ inverse Jacobi multiplier of (1) linearly independent with $V^{*}$ if and only if there exists a $C^{m-1}$ function $\phi$ which is not locally constant and such that $V=\phi(\tilde{H}) V^{*}$ on $\Omega^{*}$.

Proof. First we recall that the inverse Jacobi multiplier $V^{*}$ defined in Proposition 7 (iii) is of class $C^{m-1}$ at least and satisfies $V^{*}(t, y) \neq 0$ for any $(t, y) \in \Omega^{*}$. Second, since $H_{1}, \ldots, H_{n}$ are $C^{m}$ first integrals of (1) in $\Omega^{*}$ by hypothesis, we can define according to Definition 12 the diffeomorphism $\tilde{F}: U \rightarrow \tilde{F}(U)$ of class $C^{m}$ as $\tilde{F}(x)=\tilde{H}(0, x)$.

(i) Let $H: \Omega^{*} \rightarrow \mathbb{R}$ be a $C^{m}$ first integral of (1), take $F=H(0, \cdot)$ and $\phi=F \circ \tilde{F}^{-1}$. Then $\phi$ is $C^{m}$ and $F=\phi(\tilde{F})$ on $U$ and, further, $F(\psi(0 ; t, y))=$ $\phi(\tilde{F}(\psi(0 ; t, y)))$ for all $(t, y) \in \Omega^{*}$. But, using Proposition 7 , this last relation reads as $H=\phi(\tilde{H})$ on $\Omega^{*}$. The fact that, given some $C^{m}$ function $\phi, \phi(\tilde{H})$ is a $C^{m}$ first integral follows using Definition 6 of a first integral.

Statement (ii) follows using the previous one and Proposition 7. 


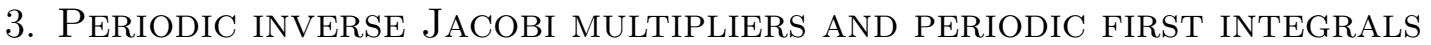

In this section we always assume what we call Hypotheses * in the Introduction. Under these hypothesis it is known that a solution $\psi(\cdot ; 0, \tilde{x}):[0, T] \rightarrow \mathbb{R}^{n}$ of system (1) satisfying $\psi(0 ; 0, \tilde{x})=\psi(T ; 0, \tilde{x})$ is actually a $T$-periodic solution, in the sense that it has an extension to $\mathbb{R}$ which is a $T$-periodic solution. Hence $\tilde{x} \in U$ is a fixed point of the Poincaré map $\Pi: U \rightarrow \tilde{U}$ if and only if $\psi(\cdot ; 0, \tilde{x})$ is a $T$-periodic solution of (1).

REMARK 15. If system (1) has a $T$-periodic solution $\psi(t ; 0, \tilde{x})$ then there exists an open neighborhood $U$ of $\tilde{x}$ that satisfies statement (c) from Hypotheses *. In order to justify this fact, one can use Lemma 6.6 and Theorem 7.6 from [1].

Let $U_{0} \subset \tilde{U}$ be open. We say that an inverse Jacobi multiplier $V$ of system (1) is $T$-periodic in $\mathbb{R} \times U_{0}$ if $V(t, x)=V(t+T, x)$ for all $t \in \mathbb{R}$ and $x \in U_{0}$. Similarly we define the $T$-periodicity of a first integral $H$ in $\mathbb{R} \times U_{0}$.

Theorem 3 stated in the Introduction shows how the existence of sufficiently many $T$-periodic and independent first integrals and nonvanishing $T$-periodic inverse Jacobi multipliers in $\mathbb{R} \times \tilde{U}$ assures the existence of continua of $T$-periodic solutions.

Proof of Theorem 3. (i) Denote $\tilde{H}=\left(H_{1}, \ldots, H_{n}\right)$ having as components the $n$ independent first integrals in $\mathbb{R} \times \tilde{U}$ that are $T$-periodic. First consider the set $\Omega^{*}$ defined by (4) in the case $U=\tilde{U}$, i.e. $\Omega^{*}=\left\{(t, \psi(t ; 0, x)): x \in \tilde{U}, t \in I_{(0, x)}\right\}$. Since $\Omega^{*} \subset \mathbb{R} \times \tilde{U}$, we also have that $H_{1}, \ldots, H_{n}$ are independent in $\Omega^{*}$. We made these considerations in order to apply Proposition 11 and deduce that $\tilde{F}: \tilde{U} \rightarrow \mathbb{R}^{n}$ defined by $\tilde{F}=\tilde{H}(0, \cdot)$ is a local diffeomorphism. We can take an open set $\hat{U} \subset U \subset \tilde{U}$, where $U$ is from Hypotheses ${ }^{*}$, such that $\tilde{x} \in \hat{U}$ and $\tilde{F}: \hat{U} \rightarrow \tilde{F}(\hat{U})$ is a diffeomorphism. We consider now the corresponding set $\hat{\Omega}^{*}$ defined by (4) for $\hat{U}$, i.e. $\hat{\Omega}^{*}=\left\{(t, \psi(t ; 0, x)): x \in \hat{U}, t \in I_{(0, x)}\right\}$ and note that we have $\hat{\Omega}^{*} \subset \Omega^{*}$. From Proposition 7 (ii) we have that $\tilde{H}(t, y)=\tilde{F}(\psi(0 ; t, y))$ for all $y \in \psi(t ; 0, \hat{U})$ and $t \in[0, T]$. We define $U_{0}=\hat{U} \cap \psi(T ; 0, \hat{U})$ and note that $U_{0}$ is an open neighborhood of $\tilde{x}$ and that both $\tilde{H}(0, y)$ and $\tilde{H}(T, y)$ are well-defined for any $y \in U_{0}$. Since $\tilde{H}(\cdot, x)$ is $T$-periodic for any $x \in \tilde{U}$, we must have $\tilde{F}(\tilde{\psi}(0 ; 0, y))=\tilde{F}(\psi(0 ; T, y))$ for any $y \in U_{0}$. From here (recalling that $\psi(0 ; 0, y)=y$ and that $\tilde{F}$ is a diffeomorphism in $\hat{U})$ it follows that $y=\psi(0 ; T, y)$ for all $y \in U_{0}$, that further gives $\psi(T ; 0, x)=x$ for all $x \in U_{0}$. This assures that $\psi(\cdot ; 0, x)$ is $T$-periodic for any $x \in U_{0}$.

(ii) Denote $H_{1}, \ldots, H_{n-1}$ the $n-1$ independent first integrals in $\mathbb{R} \times \tilde{U}$ that are $T$-periodic. In particular the gradient vectors $\nabla_{x} H_{1}(0, x), \ldots, \nabla_{x} H_{n-1}(0, x)$ are linearly independent for all $x \in \tilde{U}$ and this implies that we can select a $\mathcal{C}^{1}$ function $F_{n}: \tilde{U} \rightarrow \mathbb{R}$ to obtain $\tilde{F}: \tilde{U} \rightarrow \mathbb{R}^{n}$ defined by $\tilde{F}=\left(H_{1}(0, \cdot), \ldots, H_{n-1}(0, \cdot), F_{n}\right)$ satisfying $\operatorname{det} D \tilde{F}(x) \neq 0$ for any $x \in \tilde{U}$ (if necessary, $\tilde{U}$ can eventually be 
smaller). Like in (i), there is a sufficiently small open set $\hat{U} \subset U \subset \tilde{U}$ with $\tilde{x} \in \hat{U}$ and such that $\tilde{F}: \hat{U} \rightarrow \tilde{F}(\hat{U})$ is a diffeomorphism. Consider again $\hat{\Omega}^{*}=\left\{(t, \psi(t ; 0, x)): x \in \hat{U}, t \in I_{(0, x)}\right\}$ and $H_{n}(t, y)=F_{n}(\psi(0 ; t, y))$ for all $(t, y) \in \hat{\Omega}^{*}$. Then, if we denote $\tilde{H}=\left(H_{1}, \ldots, H_{n-1}, H_{n}\right)$ we have $\tilde{H}(t, y)=$ $\tilde{F}(\psi(0 ; t, y))$ for any $(t, y) \in \hat{\Omega}^{*}$. We define as in (i) the open neighborhood of $\tilde{x}$ given by $U_{0}=\hat{U} \cap \psi(T ; 0, \hat{U})$.

Since the first $n-1$ components of $\tilde{H}(\cdot, x)$ are $T$-periodic for any $x \in \tilde{U}$, we have that the first $n-1$ components of $\tilde{F}(y)$ and of $\tilde{F}(\psi(0 ; T, y))$ are the same for any $y \in U_{0}$. Then for each $h=\left(h_{1}, \ldots, h_{n}\right) \in \tilde{F}\left(U_{0}\right)$ the first $n-1$ components of $\tilde{F} \circ \psi(0 ; T, \cdot) \circ \tilde{F}^{-1}(h)$ are $h_{1}, \ldots, h_{n-1}$. From here we deduce that the inverse of this map shares the same property. Denote now $\tilde{U}_{0}=\tilde{F}\left(\psi\left(0 ; T, U_{0}\right)\right)$ and note that it is an open neighborhood of $\tilde{F}(\tilde{x})$. But, using properties of the inverses of compositions and recalling that the inverse of $\psi(0 ; T, \cdot)$ is $\psi(T ; 0, \cdot)$, one can see that the inverse of $\tilde{F} \circ \psi(0 ; T, \cdot) \circ \tilde{F}^{-1}: \tilde{F}\left(U_{0}\right) \rightarrow \tilde{U}_{0}$ is just $\tilde{F} \circ \psi(T ; 0, \cdot) \circ \tilde{F}^{-1}$ : $\tilde{U}_{0} \rightarrow \tilde{F}\left(U_{0}\right)$.

After all these, taking into account the definition of the Poincaré map $\Pi=$ $\psi(T ; 0, \cdot)$, we obtain that for each $h \in \tilde{U}_{0}$ the first $n-1$ components of $\tilde{F} \circ \Pi \circ$ $\tilde{F}^{-1}(h)$ are $h_{1}, \ldots, h_{n-1}$. We denote the last component of this function by $p(h)$. In this way we obtain $p: \tilde{U}_{0} \rightarrow \mathbb{R}$, which is $\mathcal{C}^{1}$, such that

$$
\tilde{F} \circ \Pi \circ \tilde{F}^{-1}(h)=\left(\begin{array}{c}
h_{1} \\
\ldots \\
h_{n-1} \\
p(h)
\end{array}\right) \quad \text { with } h=\left(\begin{array}{c}
h_{1} \\
\ldots \\
h_{n-1} \\
h_{n}
\end{array}\right) \text {. }
$$

Since $\psi(\cdot ; 0, \tilde{x})$ is $T$-periodic, we have that $\Pi(\tilde{x})=\tilde{x}$ and further, denoting

$$
\tilde{h}=\tilde{F}(\tilde{x})
$$

we obtain

$$
\tilde{F} \circ \Pi \circ \tilde{F}^{-1}(\tilde{h})=\tilde{h} .
$$

This gives $p(\tilde{h})=\tilde{h}_{n}$. We define the $\mathcal{C}^{1}$ scalar function $\tilde{p}$ of a scalar variable

$$
\mu \mapsto \tilde{p}(\mu)=p\left(\tilde{h}_{1}, \ldots, \tilde{h}_{n-1}, \mu\right)
$$

where the variable $\mu$ ranges in an open neighborhood of $\tilde{h}_{n}$. Hence we have

$$
\tilde{F} \circ \Pi \circ \tilde{F}^{-1}\left(\begin{array}{c}
\tilde{h}_{1} \\
\cdots \\
\tilde{h}_{n-1} \\
\mu
\end{array}\right)=\left(\begin{array}{c}
\tilde{h}_{1} \\
\cdots \\
\tilde{h}_{n-1} \\
\tilde{p}(\mu)
\end{array}\right)
$$

We observe that

$$
\tilde{p}\left(\tilde{h}_{n}\right)=\tilde{h}_{n}
$$

We claim that $\tilde{p}$ is the identity map in an open neighborhood of $\tilde{h}_{n}$. For any $\mu$ in that neighborhood we denote

$$
x^{*}(\mu)=\tilde{F}^{-1}\left(\tilde{h}_{1}, \ldots, \tilde{h}_{n-1}, \mu\right) .
$$


Then $\Pi\left(x^{*}(\mu)\right)=x^{*}(\mu)$ finishing the proof.

It remains to prove the claim. For this we need to use the existence of an inverse Jacobi multiplier. The hypotheses of Theorem 2 are fulfilled for the inverse Jacobi multiplier $\tilde{V}: \mathbb{R} \times \tilde{U} \rightarrow \mathbb{R}$, hence (3) is satisfied. Using that $\tilde{V}(0, x) \neq 0$ for all $x \in \tilde{U}$ and that, by $T$-periodicity of $\tilde{V}, \tilde{V}(T, \Pi(x))=\tilde{V}(0, \Pi(x))$ for any $x \in U,(3)$ gives

$$
\operatorname{det} D \Pi(x)=\frac{\tilde{V}(0, \Pi(x))}{\tilde{V}(0, x)} \quad \text { for all } x \in U .
$$

This is a fully nonlinear first order partial differential equation for the Poincaré map $\Pi$, which has as solution the identity map. Of course, when $n=1$ this reduces to a first order scalar ordinary differential equation for $\Pi$. In arbitrary dimensions, using (13), we will find that $\tilde{p}(\mu)$ satisfies a first order scalar ordinary differential equation. For this we compute the determinant of the Jacobian matrix of $\tilde{F} \circ \Pi \circ \tilde{F}^{-1}$ in two ways. First we take into account the structure of this map emphasized before, so that

$$
D\left(\tilde{F} \circ \Pi \circ \tilde{F}^{-1}\right)=\left(\begin{array}{l|l}
I_{n-1} & \\
\hline & \frac{\partial p}{\partial h_{n}}
\end{array}\right)
$$

where $I_{n}$ denotes the identity matrix of order $n$. Therefore we obtain that

$$
\operatorname{det} D\left(\tilde{F} \circ \Pi \circ \tilde{F}^{-1}\right)=\frac{\partial p}{\partial h_{n}} \text {. }
$$

Second, we use the chain rule and properties of determinants so that

$$
\operatorname{det} D\left(\tilde{F} \circ \Pi \circ \tilde{F}^{-1}\right)=\operatorname{det} D \tilde{F}\left(\Pi \circ \tilde{F}^{-1}\right) \operatorname{det} D \Pi\left(\tilde{F}^{-1}\right) \operatorname{det} D \tilde{F}^{-1} .
$$

Using the two relations above and (13) we obtain that

$$
\frac{\partial p}{\partial h_{n}}=\operatorname{det} D \tilde{F}\left(\Pi \circ \tilde{F}^{-1}\right) \frac{\tilde{V}\left(0, \Pi \circ \tilde{F}^{-1}\right)}{\tilde{V}\left(0, \tilde{F}^{-1}\right)} \operatorname{det} D \tilde{F}^{-1}
$$

holds for every variable $h \in \tilde{U}_{0}$. If we write (14) only for the vectors

$$
\tilde{h}_{\mu}=\left(\begin{array}{c}
\tilde{h}_{1} \\
\ldots \\
\tilde{h}_{n-1} \\
\mu
\end{array}\right),
$$

using (12), we obtain

$$
\frac{d \tilde{p}}{d \mu}(\mu)=\operatorname{det} D \tilde{F}\left(\tilde{F}^{-1}\left(\tilde{h}_{\tilde{p}(\mu)}\right)\right) \frac{\tilde{V}\left(0, \tilde{F}^{-1}\left(\tilde{h}_{\tilde{p}(\mu)}\right)\right)}{\tilde{V}\left(0, \tilde{F}^{-1}\left(\tilde{h}_{\mu}\right)\right)} \operatorname{det} D \tilde{F}^{-1}\left(\tilde{h}_{\mu}\right) .
$$

Hence $\tilde{p}$ is the unique solution of the Cauchy problem for the ordinary differential equation (15) with the initial condition $\tilde{p}\left(\tilde{h}_{n}\right)=\tilde{h}_{n}$. It is not difficult to see that the identity function $\tilde{p}(\mu)=\mu$, with $\mu$ in a neighborhood of $\tilde{h}_{n}$, is the solution of this initial value problem. The claim is proved. 
REMARK 16. The assumption that there exists $n-1$ independent first integrals which are $T$-periodic in Theorem 3 (ii) is essential when $n \geq 2$ as the following example shows. We consider the linear 2-dimensional system $\dot{x}=A(t) x$ with the continuous $T$-periodic in $\mathbb{R}$ diagonal matrix $A(t)=\operatorname{diag}\left\{a_{1}(t), a_{2}(t)\right\}$ such that $\int_{0}^{T} a_{i}(s) d s \neq 0$ for $i=1,2$ but $\int_{0}^{T}\left(a_{1}(s)+a_{2}(s)\right) d s=0$. This system has the $T$-periodic inverse Jacobi multiplier $V^{*}(t, x)=\exp \left(\int_{0}^{t}\left[a_{1}(s)+a_{2}(s)\right] d s\right)$ with $V^{*}(0, x)=1$, but the only $T$-periodic solution is the trivial one $\psi(t ; 0,(0,0))=$ $(0,0)$ as can be easily shown using the theory of linear systems under the conditions imposed to the coefficients of the system.

ExAMPLE 17. Let us consider the following family of systems defined in $\mathbb{R} \times \mathbb{R}^{2}$ :

$$
\dot{x}_{1}=a_{1}(t) x_{1}+x_{1}^{k_{1}} F_{1}\left(t, x_{2}\right), \quad \dot{x}_{2}=a_{2}(t) x_{2}+x_{2}^{k_{2}} F_{2}\left(t, x_{1}\right),
$$

where $k_{i} \in \mathbb{N}$, and $a_{i} \in C(\mathbb{R})$ are $T$-periodic functions satisfying $\int_{0}^{T} a_{i}(s) d s=0$. The system admits the $T$-periodic in $\mathbb{R} \times \mathbb{R}^{2}$ inverse Jacobi multiplier

$$
\tilde{V}\left(t, x_{1}, x_{2}\right)=x_{1}^{k_{1}} x_{2}^{k_{2}} \exp \left(\int_{0}^{t}\left[\left(1-k_{1}\right) a_{1}(s)+\left(1-k_{2}\right) a_{2}(s)\right] d s\right) .
$$

Hence $\tilde{V}\left(0, x_{1}, x_{2}\right) \neq 0$ if $\left(x_{1}, x_{2}\right) \in \tilde{U}=\left\{\left(x_{1}, x_{2}\right) \in \mathbb{R}^{2}: x_{1} x_{2} \neq 0\right\}$.

Notice that the axis of coordinates are invariant under the process and we have

$$
\begin{aligned}
& \psi\left(t ; 0,\left(x_{1}, 0\right)\right)=\left(x_{1} \exp \left(\int_{0}^{t} a_{1}(s) d s\right), 0\right), \\
& \psi\left(t ; 0,\left(0, x_{2}\right)\right)=\left(0, x_{2} \exp \left(\int_{0}^{t} a_{2}(s) d s\right)\right) .
\end{aligned}
$$

In particular the origin is an equilibrium and $\psi\left(t ; 0,\left(x_{1}, 0\right)\right)$ and $\psi\left(t ; 0,\left(0, x_{2}\right)\right)$ are (non-isolated) $T$-periodic solutions for each $x_{1} \in \mathbb{R}$ and $x_{2} \in \mathbb{R}$. Now the question is: what about the $T$-periodicity of $\psi\left(t ; 0,\left(x_{1}, x_{2}\right)\right)$ with $x_{1} x_{2} \neq 0$ ? Can the system have isolated $T$-periodic solutions?

To give a partial answer to this question we consider the particular case of (16),

$$
\dot{x}_{1}=a_{1}(t) x_{1}-m_{2} b(t) x_{1}^{k_{1}} x_{2}^{k_{2}-1}, \quad \dot{x}_{2}=a_{2}(t) x_{2}+m_{1} b(t) x_{1}^{k_{1}-1} x_{2}^{k_{2}},
$$

with $m_{i} \in \mathbb{N}$ and $b \in C(\mathbb{R})$. We found for (17) the $T$-periodic first integral

$$
H\left(t, x_{1}, x_{2}\right)=x_{1}^{m_{1}} x_{2}^{m_{2}} \exp \left(-\int_{0}^{t}\left[m_{1} a_{1}(s)+m_{2} a_{2}(s)\right] d s\right) \text { in } \mathbb{R} \times \mathbb{R}^{2} .
$$

One can see that $\nabla H\left(t, x_{1}, x_{2}\right) \neq(0,0,0)$ if $\left(t, x_{1}, x_{2}\right) \in \mathbb{R} \times \tilde{U}$. We intend to apply Theorem 3 (ii) in order to deduce that (17) does not have isolated $T$ periodic solutions. Let $\psi\left(t ; 0,\left(\tilde{x}_{1}, \tilde{x}_{2}\right)\right)$ be a $T$-periodic solution with $\tilde{x}_{1} \tilde{x}_{2} \neq 0$ (when $\tilde{x}_{1} \tilde{x}_{2}=0$ we already know that it is not isolated). Take $U$ an open neighborhood of $\left(\tilde{x}_{1}, \tilde{x}_{2}\right)$ with the properties guaranteed in Remark 15 . We can assume that $U \subset \tilde{U}$. We conclude now that all the hypotheses of Theorem 3 (ii) are fulfilled with $n=2$, the first integral $H$ and the inverse Jacobi multiplier $\tilde{V}$ given above. Hence, indeed the $T$-periodic solution $\psi\left(t ; 0,\left(\tilde{x}_{1}, \tilde{x}_{2}\right)\right)$ (if it exists) is not isolated in the set of $T$-periodic solutions of (17). 
An interesting particular case of system (1) is when it is divergence free, i.e. $\operatorname{div} \mathcal{X}=\sum_{i=1}^{n} \partial f_{i} / \partial x_{i}=0$ in $I \times \tilde{U}$. In this case the inverse Jacobi multiplier $V^{*}$ given in Proposition 7 (ii) is simply $V^{*}=1$, hence always $T$-periodic and non-null. Theorem 3 (ii) becomes in this case.

Corollary 18. Assume Hypothesis * and that there exists $\tilde{x} \in U$ such that $\psi(\cdot ; 0, \tilde{x})$ is a T-periodic solution of (1). Assume, in addition, that $\operatorname{div} \mathcal{X}=0$ in $I \times \tilde{U}$. If there exist $n-1$ independent first integrals in $\mathbb{R} \times \tilde{U}$ which are $T$-periodic then the T-periodic solution $\psi(\cdot ; 0, \tilde{x})$ is included into a 1-parameter family of T-periodic solutions $\psi\left(t ; 0, x^{*}(\mu)\right)$, where $x^{*}$ is a $\mathcal{C}^{1}$ function in some open interval of reals.

We restate now Theorem 3 in the particular case $n=1$, but introducing the small improvement that the conclusion holds globally. The proof does not introduce any new idea.

Theorem 19. Let $n=1$. Assume Hypothesis * and that there exists $\tilde{x} \in U$ such that $\psi(\cdot ; 0, \tilde{x})$ is a T-periodic solution of $(1)$.

(i) If there exists a first integral $H$ in $\mathbb{R} \times \tilde{U}$ of (1) which is T-periodic and such that $H(0, \cdot): U \rightarrow \mathbb{R}$ is a diffeomorphism onto its image, then $\psi(\cdot ; 0, x)$ is T-periodic for any initial condition $x \in U \cap \psi(T ; 0, U)$.

(ii) If there exist an inverse Jacobi multiplier $\tilde{V}$ in $\mathbb{R} \times \tilde{U}$ which is T-periodic and such that $\tilde{V}(0, x) \neq 0$ for all $x \in \tilde{U}$, then $\psi(\cdot ; 0, x)$ is T-periodic for any $x \in U$.

Proof. Part (i) can be easily seen following the proof of Theorem 3 (i).

(ii) In this particular case we need only relation (13) from the proof of Theorem 3 (ii), that gives that the Poincaré map $\Pi: U \rightarrow \tilde{U}$ is a solution of the ordinary differential equation

$$
\Pi^{\prime}(x)=\frac{\tilde{V}(0, \Pi(x))}{\tilde{V}(0, x)}, x \in U .
$$

In addition, we have that $\Pi(\tilde{x})=\tilde{x}$. We deduce using the uniqueness of the solution of an ordinary differential equation with $\mathcal{C}^{1}$ right-hand side, that we must have $\Pi(x)=x$ for any $x \in U$.

REMARK 20. The assumption that there exists a $T$-periodic solution $\psi(\cdot ; 0, \tilde{x})$ with $\tilde{x} \in U$ is essential in Theorem 19 (i) as the following example shows. We consider the scalar ordinary differential equation $\dot{x}=x$, with $I=\mathbb{R}, \tilde{U}=(0, \infty)$ and $U=(1,2)$. One can check that $H(t, x)=\sin (t-\ln x)$ is a $2 \pi$-periodic first integral in $\mathbb{R} \times \tilde{U}$ of $\dot{x}=x$. Moreover, $H(0, x)=-\sin (\ln x)$ is a diffeomorphism from $U=(1,2)$ onto its image. But it is easy to see that none of the solutions $\psi(\cdot ; 0, x)$ with $x \in U$ is $2 \pi$-periodic. In fact here we have that $U \cap \psi(2 \pi ; 0, U)=$ $(1,2) \cap\left(e^{2 \pi}, 2 e^{2 \pi}\right)=\emptyset$. 
REMARK 21. The assumption that there exists a $T$-periodic solution $\psi(\cdot ; 0, \tilde{x})$ with $\tilde{x} \in U$ is essential in Theorem 19 (ii) as the following example shows. We consider the scalar linear ordinary differential equation $\dot{x}=a(t) x+b(t)$, where $a, b$ are continuous and $T$-periodic scalar functions. Assume that $\int_{0}^{T} a(s) d s=0$ and $\int_{0}^{T} \exp \left(-\int_{0}^{t} a(s) d s\right) b(t) d t \neq 0$. In these conditions this linear differential equation has no $T$-periodic solution. Take $I=\tilde{U}=U=\mathbb{R}$. One can check that $\tilde{V}(t, x)=V^{*}(t, x)=\exp \left(\int_{0}^{t} a(s) d s\right)$ is a $T$-periodic inverse Jacobi multiplier in $\mathbb{R} \times \mathbb{R}$ that also satisfies $\tilde{V}(0, x)=1 \neq 0$.

REMARK 22. The assumption that $\tilde{V}(0, x) \neq 0$ for all $x \in \tilde{U}$ is essential in Theorem 19 (ii) as the following example shows. We consider again the scalar ordinary differential equation $\dot{x}=x$, this time with $I=\tilde{U}=U=\mathbb{R}$ and $T>0$ arbitrary fixed. We have that the solution $\psi(t ; 0,0)=0$ is $T$-periodic and the inverse Jacobi multiplier $V(t, x)=x$ is also $T$-periodic in $\mathbb{R} \times \mathbb{R}$. But there are no other $T$-periodic solutions of $\dot{x}=x$.

We are interested now to study the reciprocal of Theorem 3 (i). The next Lemma and Theorem state in what conditions is valid.

Lemma 23. Assume Hypothesis * and suppose that $\psi(\cdot ; 0, x)$ is T-periodic for all $x \in U$. Let $H: \Omega^{*} \rightarrow \mathbb{R}$ and $V: \Omega^{*} \rightarrow \mathbb{R}$ be a first integral, and, respectively, an inverse Jacobi multiplier of (1). Then

$$
H(t+T, y)=H(t, y) \quad \text { and } \quad V(t+T, y)=V(t, y) \quad \text { for all } \quad(t, y) \in \Omega^{*} .
$$

If there exists an open set $U_{0} \subset U$ such that $\mathbb{R} \times U_{0} \subset \Omega^{*}$ then both $H$ and $V$ are $T$-periodic in $\mathbb{R} \times U_{0}$.

Proof. Let $(t, y) \in \Omega^{*}$, and denote $x=\psi(0 ; t, y) \in U$. Then $y=\psi(t ; 0, x)=$ $\psi(t+T ; 0, x)$ and, further, $x=\psi(0 ; t+T, y)$. Thus, using the hypothesis that $\psi(\cdot ; 0, x)$ is $T$-periodic for any $x \in U$, we have proved that

$$
\psi(0 ; t+T, y)=\psi(0 ; t, y) \text { for any }(t, y) \in \Omega^{*} .
$$

Now, the $T$-periodicity of $\psi(\cdot ; 0, x)$ for any $x \in U$ assures also the $T$-periodicity of $D_{x} \psi(\cdot ; 0, x)$ for any $x \in U$. This fact and relation (18) assure that $V^{*}(t, y)=$ $\operatorname{det}\left(D_{x} \psi(t ; 0, \psi(0 ; t, y))\right)$ that appears in Proposition 7 satisfies

$$
V^{*}(t+T, y)=V^{*}(t, y) \text { for any }(t, y) \in \Omega^{*} .
$$

The conclusion follows using the expressions of $H$ and $V$ given in Proposition 7 and relations (18) and (19).

Theorem 24. Assume Hypothesis * and suppose that $\psi(\cdot ; 0, x)$ is T-periodic for all $x \in U$. Fix $\tilde{x} \in U$ and denote $\varphi=\psi(\cdot ; 0, \tilde{x})$. Let $H: \Omega^{*} \rightarrow \mathbb{R}$ and $V: \Omega^{*} \rightarrow \mathbb{R}$ be a first integral, and, respectively, an inverse Jacobi multiplier of (1). Then there exists an open neighborhood $U_{0} \subset U$ of $\tilde{x}$ such that

$$
H(t+T, y+\varphi(t))=H(t, y+\varphi(t)) \quad \text { and } \quad V(t+T, y+\varphi(t))=V(t, y+\varphi(t))
$$


for all $t \in \mathbb{R}$ and $y \in U_{0}$. In particular, if $\psi(t ; 0, \tilde{x})=\tilde{x}$ for all $t \in \mathbb{R}$ then $H$ and $V$ are $T$-periodic in $\mathbb{R} \times U_{0}$.

Proof. We consider first the particular case that the fixed solution is the null solution, i.e. $\psi(t ; 0,0)=0$ for all $t \in \mathbb{R}$. In this case, for any $t \in[0, T]$ we have that $\psi(t ; 0, U)$ is an open neighborhood of 0 , which implies that

$$
U_{0}=\bigcap_{t \in[0, T]} \psi(t ; 0, U) \subset U
$$

is an open neighborhood of 0 . Since $\left.\Omega^{*}=\{(t, \psi(t ; 0, x)): t \in \mathbb{R}, x \in U)\right\}$ and $\psi(\cdot ; 0, x)$ is $T$-periodic for all $x \in U$, from the definition of $U_{0}$ it is clear that $\mathbb{R} \times U_{0} \subset \Omega^{*}$. The conclusion follows by Lemma 23 .

Consider now the general case and let $H$ and $V$ like in the hypothesis. We introduce the new variable $u=x-\varphi(t)$ so that equation (1) is transformed into equation

$$
\dot{u}=f(t, u+\varphi(t))-f(t, \varphi(t)) .
$$

Its process $\gamma$ satisfies $\gamma(t ; 0 ; u)=\psi(t ; 0, u+\tilde{x})-\varphi(t)$ for all $u \in U-\tilde{x}$, assuring that $\gamma(\cdot ; 0, u)$ is $T$-periodic for all $u \in U-\tilde{x}$ and that $\gamma(t ; 0,0)=0$ for all $t \in \mathbb{R}$. Then equation $(21)$ is in the particular case solved before. It can be easily shown that $H(t, u+\varphi(t))$ and $V(t, u+\varphi(t))$ is a first integral, and, respectively, an inverse Jacobi multiplier of (21). Thus they are $T$-periodic in $\mathbb{R} \times U_{0}$. The proof is done.

\section{REFERENCES}

[1] H. Amann, Ordinary Differential Equations. An Introduction to Nonlinear Analysis, de Gruyter Studies in Mathematics, 13 Walter de Gruyter \& Co., Berlin, 1990.

[2] L.R. Berrone and H. Giacomini, Inverse Jacobi multipliers, Rend. Circ. Mat. Palermo (2) 52 (2003), 77-130.

[3] A. Buică, I. A. García And S. Maza, Existence of inverse Jacobi multipliers around Hopf points in $\mathbb{R}^{3}$ : emphasis on the center problem, J. Differential Equations 252 (2012), 6324-6336.

[4] A. Buiç̆, I. A. García, S. Maza, Multiple Hopf bifurcation in $\mathbb{R}^{3}$ and inverse Jacobi multipliers, J. Differential Equations 256 (2014), 310-325.

[5] C. Chicone, Ordinary Differential Equations with Applications. (2 ed.), New York: Springer-Verlag, 2006.

[6] A. Enciso And D. Peralta-Salas, Existence and vanishing set of inverse integrating factors for analytic vector fields, Bull. London Math. Soc. 41 (2009), 1112-1124.

[7] R. Flores-Espinoza, Periodic first integrals for Hamiltonian systems of Lie type, Int. J. Geom. Meth. Mod. Phys. 8 (2011), 1169-1177.

[8] I.A. García And M. Grau, A survey on the inverse integrating factor, Qual. Theory Dyn. Syst. 9 (2010), 115-166.

[9] I.A. García, H. Giacomini and M. Grau, The inverse integrating factor and the Poincaré map, Trans. Amer. Math. Soc. 362 (2010), 3591-3612.

[10] I.A. García, H. Giacomini and M. Grau, Generalized Hopf bifurcation for planar vector fields via the inverse integrating factor, J. Dyn. Differ. Equ. 23 (2011), 251-281.

[11] J.K. HALE, Ordinary Differential Equations, 2nd Edition, Robert E. Krieger Publishing Co., Huntington, NY, 1980. 
[12] C.G.J. JACOBI, Sul principio dellultimo moltiplicatore, e suo uso come nuovo principio generale di meccanica, Giornale Arcadico di Scienze, Lettere ed Arti 99 (1844), 129-146.

[13] P.E. Klooeden and M. Rasmussen, Nonautonomous Dynamical Systems, Mathematical Surveys and Monographs 176, American Mathematical Society, 2011.

[14] X. ZHANG, Inverse Jacobian multipliers and Hopf bifurcation on center manifolds, J. Differential Equations 256 (2014), 3552-3567.

${ }^{1}$ Department of Mathematics, Babeş-Bolyai University, Kogălniceanu 1, 400084 Cluj-Napoca, Romania

E-mail address: abuica@math.ubbcluj.ro

2 Departament de Matemàtica, Universitat de Lleida, Avda. Jaume II, 69, 25001 Lleida, Spain

E-mail address: garcia@matematica.udl.cat 